Swarthmore College

Works

5-1-2013

\title{
Modeling Of Microbubbles Pushed Through Clots Via Acoustic Radiation Force
}

\author{
E. Carr Everbach \\ Swarthmore College, ceverba1@swarthmore.edu \\ Ascanio Guarini , '16
}

Follow this and additional works at: https://works.swarthmore.edu/fac-engineering

Part of the Engineering Commons

Let us know how access to these works benefits you

\section{Recommended Citation}

E. Carr Everbach and Ascanio Guarini , '16. (2013). "Modeling Of Microbubbles Pushed Through Clots Via Acoustic Radiation Force". Proceedings Of Meetings On Acoustics. Volume 19, DOI: 10.1121/1.4800645 https://works.swarthmore.edu/fac-engineering/106

This work is brought to you for free by Swarthmore College Libraries' Works. It has been accepted for inclusion in Engineering Faculty Works by an authorized administrator of Works. For more information, please contact myworks@swarthmore.edu. 
Modeling of microbubbles pushed through clots via acoustic radiation force

E. C. Everbach, and Ascanio Guarini

Citation: Proc. Mtgs. Acoust. 19, 075044 (2013);

View online: https://doi.org/10.1121/1.4800645

View Table of Contents: http://asa.scitation.org/toc/pma/19/1

Published by the Acoustical Society of America 


\title{
Proceedings of Meetings on Acoustics
}
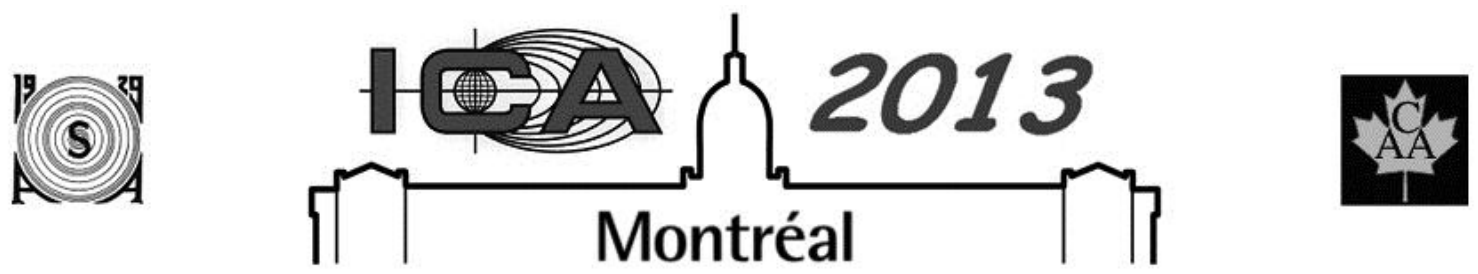

\author{
ICA 2013 Montreal \\ Montreal, Canada
}

2 - 7 June 2013

Biomedical Acoustics

Session 2pBAa: Bubbles Bubbles Everywhere II

\section{2pBAa8. Modeling of microbubbles pushed through clots via acoustic radiation force}

E. C. Everbach* and Ascanio Guarini

*Corresponding author's address: Engineering, Swarthmore College, 500 College Avenue, Swarthmore, PA 19081, ceverba1@swarthmore.edu

Previous studies have shown that thrombi, which may completely block the blood flow in a vessel, can be dissolved by ultrasound acting on echo-contrast agent microbubbles. The presumed mechanism is acoustic cavitation, the radial oscillations of the bubbles, which can exert locally large forces on the fibrin ropes that make up the clot matrix. However, the movement of the bubbles through the clot in the absence of flow suggests that acoustic radiation force also plays an important role. Because detailed mechanistic modeling of this process is not available, we present here a heuristic study in which microbubble transit times in gels of various porosities were measured and described by a simplified percolation theory. Results suggest considerations for optimizing the penetration of active microbubbles in sonothrombolysis.

Published by the Acoustical Society of America through the American Institute of Physics 


\section{Introduction}

Sonothrombolysis is the acceleration of blood clot dissolution with applied ultrasound, first reported by Tachibana in $1992^{1}$, who noted increased effectiveness of recombinant tissue-plasminogen activator (rtPA) in dissolving thrombi that were being visualized by ultrasound ${ }^{2}$. Also sometimes called ultrasound-accelerated thrombolysis, the mechanism of this acceleration was thought initially to be simple heating, which drives the chemical reactions of enzymatic thrombolysis faster. However, research with low-duty-cycle ultrasound to avoid bulk heating still showed an enhanced effect of ultrasound ${ }^{3}$. Investigations into clot microscructure further showed that ultrasound exposure changed the distribution of mean diameters of the fibrin strands that make up the clot structure $^{4}$. Overpressure studies showed that microbubbles present in the clot account for over half of the acceleration effect, implicating acoustic cavitation as a dominant (but not the sole) mechanism ${ }^{5}$. Thereafter, research focused efforts largely focused on how endogenous microbubbles could be introduced into clot systems in vitro $^{6}$ and in vivo ${ }^{78}$. Interestingly, the naturally-occurring clot lysing enzymes present in vivo allow effective thrombolysis to occur without the addition of rtPA or streptokinase.

Much recent work has involved detection and monitoring of cavitational activity during sonothrombolysis, especially distinguishing between stable cavitation and inertial cavitation. Claims of superior clot destruction attending stable cavitation but not inertial cavitation have suggested the possibility of an optimum cavitational regime, which would minimize treatment time for a given percentage of clot dissolution. Thus efforts are underway to develop so-called "theranostic" ultrasound devices that would insonify and monitor cavitational activity to maximize efficacy and minimize undesirable bioeffects. Central to these efforts is a model of bubble activity that can be used to guide the real-time algorithms governing the performance of such devices.

While single-bubble numerical models have been successful in describing the behavior of dilute suspensions of echo-contrast agents in buffer solution and blood, multi-bubble cavitational models that take into account bubblebubble interactions have yet to be perfected. Typical dilutions used in experiments are in the range 1-5 x 107 microbubbles/ul, so that mean bubble-bubble distances are on the order of ten bubble diameters. Moreover, clinical echo-contrast agents are not a monodisperse population as they come from the manufacturer, but rather have a size distribution which is further shifted in unknown ways once diluted or injected in vivo. Therefore multi-bubble models must take into account the different resonance frequencies of the different-sized bubbles, whose spatial distribution may determine meta-resonance or bubble-cloud effects.

The present study investigates bubble translational, as opposed to radial, motion. Acoustic radiation force acting on a bubble has been studied previously both theoretically and experimentally as a fundamentally nonlinear phenomenon. As a second-order force, arising from the deposition of momentum in a bubble due to its acoustic impedance mismatch with the surrounding liquid, acoustic radiation force from an ultrasonic traveling wave acts to push the bubble away from the transducer and in the direction of the wave vector. This force is a strong function of wave amplitude and is particularly large for bubbles of resonance frequency. In an unconfined liquid, spherical bubbles will quickly accelerate to a terminal velocity whose value is determined by a balance of acoustic radiation force and viscous drag. An additional complication is that resonant bubbles will be undergoing maximum radial excursions during the translation, which conservation of momentum dictates will accelerate and de-accelerate relative to their terminal velocity.

In a blood clot, the propagation path for translating bubbles is not open, but rather blocked by fibrin ropes that make up the clot matrix. While the precise interaction between bubbles and clot fibers is not known, for bubbles of resonant size at $1 \mathrm{MHz}$, the bubble size and mean pore spacing between fibers are of the same order. Thus it may be that bubbles are driven into pores where they are trapped until a succeeding compressive half-cycle of the waves decreases their radii sufficiently to allow them to penetrate into the succeeding pore. An interesting question, not addressed by this investigation, is whether bubble growth during the rarefactional acoustic half-cycle allows the bubbles to pry apart clot fibers, and what effect such stresses in the fibrin ropes have to weaken them or expose potential binding sites to thrombolytic enzyme molecules. A comprehensive model of this process is the ultimate goal of the investigators, but a simpler model may be adequate to start.

\section{Percolation Theory Applied to Single-Bubble Clot-Matrix Interactions}

Percolation theory addresses questions of the probability that a path can be found between two points in a domain ${ }^{9}$. Originally developed for two-dimensional computational domains, as the number of impenetrable 
elements are added randomly, the probability that such a path connecting widely-separated points drops precipitously at a critical density, which appears to be a universal value ${ }^{10}$. Three-dimensional domains have also been considered, with similar results ${ }^{11,12}$.

As a start, a computational model of the children's game "Kerplunk" can be viewed as analogous to a singlebubble interaction with clot fibers. In this model, the bubble does not change diameter within an acoustic period but acts as a solid sphere moving through a lattice of straight rods at a constant terminal velocity. When the sphere encounters a rod, it moves as a marble would under gravity in Kerplunk, falling to the side of the rod on which its centroid is located. For perfectly straight, rigid fibers of infinitesimal diameter, the sphere would follow a serpentine path until it reached the anterior face of the clot, and exit into open liquid where it would move in a straight line. As the density of fibers crossing the domain is increased, the probability that a sphere released from one face of the clot would reach the other and exit diminishes precipitously. The relative size difference between sphere diameter and mean spacing between rods is an important parameter. To provide input data for this developing model, a series of experiments were conducted to measure the time taken for bubbles with an estimated size distribution to pass through gels of varying porosities as a function of ultrasonic pulse parameters.

According to classical radiation force theory ${ }^{13}$, the force exerted on a spherical bubble of equilibrium radius $\mathrm{R}_{0}$ by a plane harmonic wave of angular frequency $\omega$ is given by:

$$
F_{\text {rad }}=\pi \rho_{0}|A|^{2} k_{0} R_{0} \frac{\left(\frac{\omega_{0}}{\omega}\right)^{2}\left(\frac{\delta_{v}}{R_{0}}\right)^{2}}{\left(\frac{\omega_{0}}{\omega}\right)^{4}+4\left(\frac{\delta_{v}}{R_{0}}\right)^{4}}
$$

where $\rho_{0}$ is the fluid density, $\omega_{0}$ is the bubble resonance frequency, $A$ is the particle displacement amplitude and $\mathrm{k}_{0}$ the wavenumber, and $\delta_{v}$ is the viscous penetration depth in the liquid surrounding the bubble. This force acts in a direction away from the transducer (for traveling waves). Acting in the opposite direction is the Stokes drag on a moving spherical bubble:

$$
F_{\text {drag }}=4 \pi \mu V R_{0}
$$

where $\mu$ is the liquid shear viscosity and $V$ is the bubble velocity relative to the surrounding liquid. As the bubble accelerates, the two forces balance and the bubble moves at a constant terminal velocity in a direction away from the transducer $^{14}$.

\section{METHODS}

Gelatin "clots" of varying porosity $(1 \%, 2 \%$, and $5 \%)$ were produced by dissolving Knox ${ }^{\mathrm{TM}}$ unflavored gelatin in boiling distilled water and allowing 12 hours for the gels to set at $20^{\circ} \mathrm{C}$ and $50 \%$ relative humidity. Sample holders were fabricated as follows. 5-cm-long straight sections of hollow nylon tubing of $3 \mathrm{~mm}$ inner diameter and $0.5 \mathrm{~mm}$ wall thickness were carved with a dremel rotating bit to expose a central portion as shown in Figure 1. A 3-mmdiameter hole was also drilled in the tubing section in the back of the tube and brass rods inserted at right angles to form a "T." This assembly was placed in a silicone rubber mold that was filled with 10\%-by-weight gelatin solution prepared as above. Once the gelatin had set, the rods were removed and the leg of the " $\mathrm{T}$ " filled with the sample gelatin (shown pink in Figure 1), leaving the top of the "T" empty as a conduit for a flowing medium. The result was a T-shaped sample holder carrying a flowing solution consisting of $0.9 \%$ saline, diluted india ink, and Definity ${ }^{\mathrm{TM}}$ microbubbles (in varying dilutions). The gelatin "clots" were formed in the vertical portion of the "T," which was aligned with the ultrasonic beam in a water tank, as shown in Figure 2. Thus microbubbles carried across the "T" are pushed into the proximal end of the cylindrical "clot" and exit through the anterior end of the clot at the foot of the $\mathrm{T}$ and into the water tank. This arrangement is similar to that of previous in vitro studies.

\section{$20 \mathrm{MHz}$ Passive Cavitation Detector}

The gelatin sample holder allows passage of $1.1 \mathrm{MHz}$ tone bursts of $1.7 \mathrm{MPa}$ peak rarefactional pressure and 80 acoustic periods through the gelatin "clot" at a pulse repetition frequency (PRF) of $10 \mathrm{~Hz}$. These parameters were found in preliminary testing to cause inertial cavitation of bubbles exiting the clot. Inertial cavitation was measured 
by a single-crystal lithium niobate $20 \mathrm{MHz}$ Passive Cavitation Detector (PCD; UTX Inc. Holmes, NY USA) whose focal diameter was $1.4 \mathrm{~mm}$ in radius and $5 \mathrm{~mm}$ in length, placed at right angles to the primary $1.1 \mathrm{MHz}$ axis and aligned with it. The PCD's output was passed through a fourth-order Butterworth bandpass filter of center frequency $20.5 \mathrm{MHz}$ and $0.5 \mathrm{MHz}$ bandwidth, amplified, and displayed on a digital oscilloscope (LeCroy Waverunner, Chestnut Ridge, NY USA). The PCD therefore recorded the $20 \mathrm{MHz}$ components of broad-band noise accompanying the inertial collapse of Definity ${ }^{\mathrm{TM}}$ microbubbles exiting the foot of the T sample holder. The peakto-peak and root-mean-square voltages of signals appearing on the PCD oscilloscope were recorded with each ultrasonic tone burst, and logged to computer memory for later plotting.

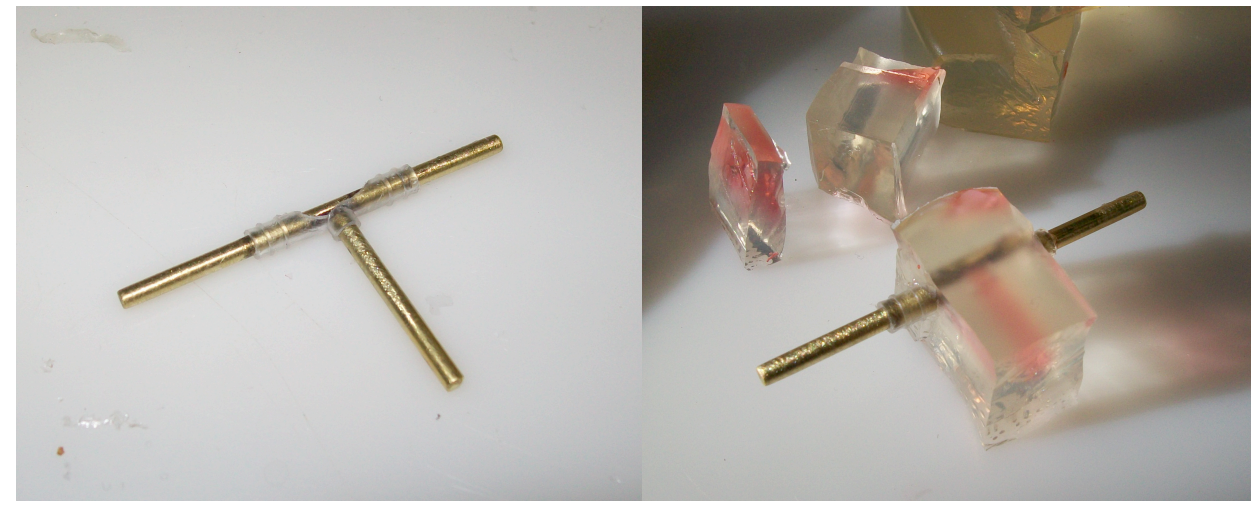

FIGURE 1A AND 1B. Fabrication of sample holders shown, in which $10 \%$ gelatin surrounds a T-shaped channel. The top part of the "T" is formed via a straight length of tubing with a brass rod inserted through it (shown). The descending leg of the " $T$ " is formed by another brass rod. Following gel setting in a mold, the brass rods are removed and the sample is formed in the leg (shown as pink).

A Lucite ${ }^{\mathrm{TM}}$ tank of dimensions $26 \mathrm{~cm}$ (L) by $22 \mathrm{~cm}$ (W) by $16 \mathrm{~cm}$ (D) was filled with distilled, degassed water and maintained at $20^{\circ} \mathrm{C}$. Rigidly mounted in the wall of the tank was a $1.1 \mathrm{MHz}$ transducer consisting of a $2.5-\mathrm{cm}-$ diameter disk of PZT-4 bonded to an aluminum lens with an 11.4-cm focal depth. The $6 \mathrm{~dB}$ focal envelope was measured to be $3.1 \mathrm{~mm}$ in diameter and $8.4 \mathrm{~mm}$ long using a $0.2 \mathrm{~mm}$-diameter PVDF hydrophone (Precison Acoustics, Dorset UK). A computer-controlled 3-axis positioner (Velmex Unislide, Bloomfield, NY USA) was able to place the sample holder so that the gelatin "clot" was located within the focal area. The $20 \mathrm{MHz}$ PCD was positioned confocally with the co-focal region located $1 \mathrm{~mm}$ from the anterior clot face to serve as a detector of inertial cavitation of bubbles exiting the clot.

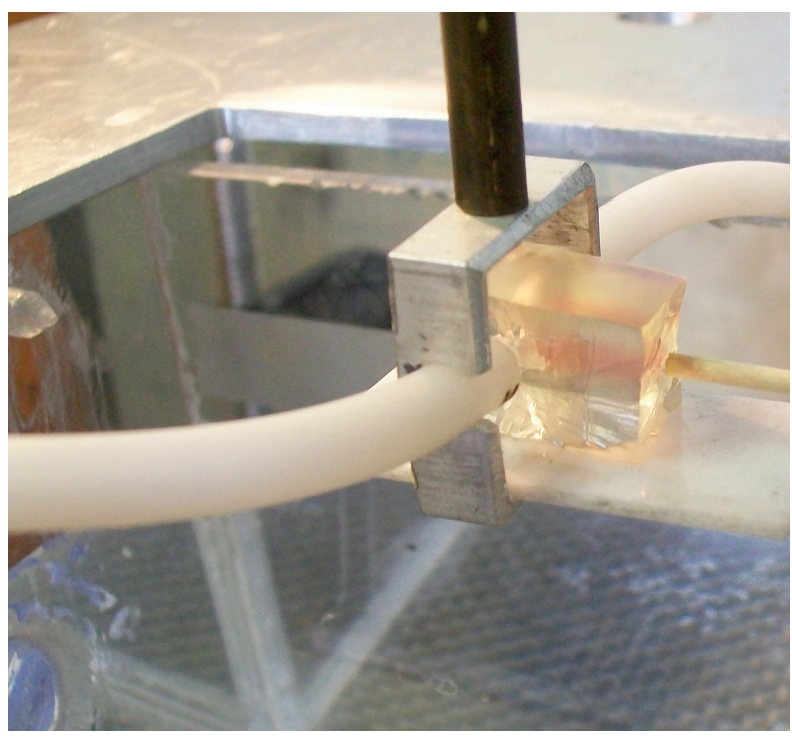


FIGURE 2. Sample shown mounted on three-axis positioner with connecting tubing carrying bubbly liquid. A $1.1 \mathrm{MHz}$ transducer produces focused traveling waves along the axis of the sample. Microbubbles are pushed by acoustic radiation force into and through the sample (shown pink) and exit into the water tank at the point shown by the tip of the white stick (shown as position indicator).

One $0.5 \mathrm{~mL}$ vial of Definity ${ }^{\mathrm{TM}}$ microbubbles (Lantheus Medical Imaging, North Billerica, MA USA) and $0.1 \mathrm{~mL}$ India ink (for flow visualization) were added to $100 \mathrm{~mL}$ normal $(0.9 \%)$ saline and this solution pushed via syringe pump through the top portion of the "T" sample holder at a rate of $5 \mathrm{~mL} / \mathrm{min}$. With an estimated bubble concentration of $60 \times 10^{6}$ microbubbles $/ \mathrm{mL}$ in the size range $1.1-3.3 \mu \mathrm{m}^{15}$ and a PRF of $10 \mathrm{~Hz}$, approximately 500 000 microbubbles were flowing past the anterior face of the cylindrical "clot" with each toneburst.

\section{RESULTS}

With no microbubbles circulating, the PCD output was indistinguishable from background electrical noise with or without ultrasound. When Definity ${ }^{\mathrm{TM}}$ microbubbles in concentrations greater than $100 \mu \mathrm{L} / \mathrm{mL}$ were circulated through the top of the sample holder, cavitational activity as measured by PCD output appeared after several seconds following transducer activation. Table 1 shows the onset delay from transducer activation to first PCD activity for three densities of gelatin samples, each $5 \mathrm{~mm}$ long.

TABLE 1. Time delay between transducer activation and inertial cavitation detection for $5 \mathrm{~mm}$ long gelatin clot

\begin{tabular}{ccc}
\hline Gelatin "clot" density (g/g H $\mathbf{2} \mathbf{O})$ & onset delay mean \pm stdev (s) & number of samples \\
\hline $1 \%$ & $5.5 \pm 1.2$ & 14 \\
$2 \%$ & $5.8 \pm 0.9$ & 18 \\
$5 \%$ & $8.3 \pm 1.4$ & 12 \\
\hline
\end{tabular}

If the onset delay times are correctly interpreted as the transit time for bubbles to cross from one end of the cylindrical sample to the exit, then bubble velocities through the clot are approximately $0.91,0.86$, and $0.60 \mathrm{~mm} / \mathrm{s}$, respectively, for $1 \%, 2 \%$, and $5 \%$ gels. According to calculations of microbubble terminal velocities at the parameters used in this study, bubbles should be moving on the order of $10-30 \mathrm{~mm} / \mathrm{s}$, depending on diameter.

\section{DISCUSSION}

Definity ${ }^{\mathrm{TM}}$ microbubbles may be pushed by acoustic radiation force through a gelatin plug, where they emerge into the unbounded volume of a water tank and undergo inertial cavitation. The gelatin plug offers a simplified model of a fibrin clot. Microbubble movement through the clot is one order of magnitude slower than would be expected if the bubbles were in an unconstrained medium. What remains is to develop a numerical model, based on percolation theory and Monte Carlo simulation of the children's game Kerplunk ${ }^{\mathrm{TM}}$, that propagates a virtual sphere through a medium of virtual rods. Parameters of the simulation may be matched to measured results from the bubble-gelatin experiment. The authors hope to be able to report success in connecting these modeling efforts with the measured data.

\section{ACKNOWLEDGMENTS}

The authors were funded through a grant from the Howard Hughes Medical Institute awarded to Swarthmore College. Guidance in Monte Carlo simulation of percolation theory was graciously provided by Professors Lynne Schofield and Matthew Sedlock of Swarthmore's Department of Mathematics and Statistics. 


\section{REFERENCES}

\footnotetext{
${ }^{1}$ K. Tachibana, "Enhancement of fibrinolysis with ultrasound energy," JVIR 3, 299-303 (1992).

${ }^{2} \mathrm{~K}$. Tachibana and S. Tachibana, "Albumin microbubble echo-contrast material as an enhancer for ultrasound accelerated thrombolysis," Circulation 92, 1148-1150 (1995).

${ }^{3}$ C.W. Francis, P.T. Onundarson, E.L. Carstensen, A. Blinc, and R.S. Meltzer, "Enhancement of fibrinolysis in vitro by ultrasound," J. Clin. Invest. 90, 2063-2068 (1992).

${ }^{4}$ A. Blinc, C.W. Francis, J.L. Trudnowski, and E.L. Carstensen, "Characterization of ultrasound-potentiated fibinolysis in vitro," Blood 81, 2636-2643 (1993).

${ }^{5}$ E.C. Everbach and C.W. Francis, "Cavitational mechanisms in ultrasound-accelerated thrombolysis at $1 \mathrm{MHz}$," Ultrasound in Med. Biol. 26(7), 1153-1160 (2000).

${ }^{6}$ F. Xie, E.C. Everbach, S. Gao, L.K. Drvol, W.T. Shi, F. Vignon, J.E. Powers, J. Lof, T.R. Porter, "Effects of attenuation and thrombus age on the success of ultrasound and microbubble-mediated thrombus dissolution," Ultrasound in Med. \& Biol. 37(2), 280-288 (2011).

${ }^{7}$ W.T. Shi, T.R. Porter, F. Vignon, J.E. Powers, S. Gao, J. Liu, F.Xie, L.Drvol, J.Lof, and E.C. Everbach, "Investigation of image-guided sonothrombolysis in a porcine acute ischemic stroke model," IEEE Ultrasonics 2011, 332-337.

8 F. Vignon, W. Shi, J. Liu, F. Xie, S. Gao, L. Drvol, J. Lof, C. Everbach, T. Porter, and J. Powers, "In-vivo microbubble cavitation imaging." Proceedings of the Twelfth International Symposium on Therapeutic Ultrasound, 2011, 134-38.

${ }^{9}$ M.E.J. Newman and R.M. Ziff, "Fast Monte Carlo algorithm for site or bond percolation," Phys. Rev. E 64, 016706 (2001).

${ }^{10}$ Y.B. Yi and K. Esmail, "Computational measurement of void percolation thresholds of oblate particles and thin plate composites," J. Appl. Phys. 111(2), 124903 (2012).

${ }^{11}$ Y.B. Yi, "Void percolation and conduction of overlapping ellipsoids," Phys. Rev. E 74, 031112 (2006).

${ }^{12}$ W.T. Elam, A.R. Kerstein, and J.J. Rehr, "Critical properties of the void percolation problem for spheres," Phys. Review Lett. 52(17), 1516-1519 (1984).

${ }^{13}$ A.A. Doinikov, "Acoustic radiation force on a bubble: viscous and thermal effects,” J. Acoust. Soc. Am. 103(1), 143-147 (1998).

${ }^{14}$ X. Yang and C.C. Church, "A model for the dynamics of gas bubbles in soft tissue,” J. Acoust. Soc. Am. 118(6), 3595-3606 (2005).

${ }^{15}$ D.E. Goertz, N. de Jong, and A.F.W. van der Steen, "High frequency attenuation and size distribution measurements of Definity and manipulated definity populations," IEEE Ultrason. Symp. 1051-0117, 1552-1555 (2006).
} 